\title{
LA DIMENSIÓN GEOPOLÍTICA DEL INTERÉS ESTATAL: EL ATLÁNTICO SUR OCCIDENTAL Y SU RELEVANCIA PARAARGENTINA
}

\author{
THE GEOPOLITICAL DIMENSION OF THE STATE INTEREST: \\ THE WESTERN SOUTH ATLANTIC AND \\ ITS RELEVANCE FOR ARGENTINA
}

\author{
Ezequiel Magnani ${ }^{1}$ \\ ORCID: 0000-0002-6099-9325
}

\section{RESUMEN}

¿Qué factores hacen que los Estados les den relevancia geopolítica a determinados espacios geográficos? ¿Cuál es la importancia estratégica que la Argentina le otorga a la región del Atlántico Sur Occidental? Para responder estas interrogantes, en el presente artículo se realiza un repaso de las principales tradiciones geopolíticas para identificar aquellos factores que hacen que un determinado espacio geográfico se vuelva parte del interés de un Estado. Luego, mediante un estudio de caso cualitativo se realiza un análisis geopolítico del Atlántico Sur Occidental mediante documentos oficiales del Ministerio de Defensa y del Ministerio de Relaciones Exteriores y Culto de la Argentina. A partir de ello, se concluye que la presencia de factores geopolíticos tales como el potencial económico de la región, las disputas de soberanía allí presentes y la incertidumbre en lo que respecta tanto a la continuidad del Tratado Antártico y el Protocolo de Madrid como a la posibilidad de futuros escenarios adversos, hacen que el Estado argentino preste una considerable atención a la región del Atlántico Sur Occidental a la hora de establecer sus objetivos y prioridades en materia de defensa nacional y política exterior.

Palabras clave: Argentina; Geopolítica; Atlántico Sur; Seguridad; Defensa; Interés nacional

\begin{abstract}
What factors cause states to give geopolitical relevance to certain geographical areas? What is the strategic importance that Argentina gives to the Western South Atlantic? To answer the questions, this article reviews the main geopolitical traditions in order to identify those factors that make a geographical space relevant for the interest of a determined State. Then, is conducted out a qualitative case study in order to elaborate, in the first place, a geopolitical analysis of the Western South Atlantic thought the analysis of official documents of the Ministry of Defense and the Ministry of Foreign Affairs of Argentina. Considering this, we conclude that the presence of geopolitical factors such as the economic potential of the region, the sovereignty disputes and the uncertainty regarding the continuity of the Antarctic Treaty and the Madrid Protocol as well as the possible future adverse scenarios, make the Argentinean State pay considerable attention to the Western South Atlantic region when establishing its objectives and priorities of foreign policy and national defense.
\end{abstract}

Keywords: Argentina; Geopolitics; South Atlantic; Security; Defense; National Interest

1 Universidad Torcuato Di Tella, Departamento de Ciencia Política y Estudios Internacionales, Buenos Aires, Argentina. Profesor asistente, candidato a Doctor en Estudios Internacionales y director de la comisión de Defensa y Seguridad Internacional de la Fundación Meridiano. Correo electrónico: ezequielmagnani11@gmail.com 


\section{Introducción}

Desde los primeros escritos sobre geopolítica se puede identificar una conexión fuerte e implícita entre la geografía y el interés de los Estados. La mayoría de los geopolíticos buscaban analizar y dar cuenta de la realidad geopolítica de su región para luego formular, de manera propositiva, las políticas que su Estado debería llevar adelante para poder cumplir con sus supuestos intereses. En esta línea, los geopolíticos argentinos han demostrado a lo largo de los años un marcado interés por la región del Atlántico Sur Occidental y su relevancia para la Argentina (Assef, 1980; Guglialmelli, 1979; Hernández y Chitarroni, 1977; Storni, 1967). En consecuencia, han realizado prolíficos análisis sobre las implicancias de dicha región para el país, y gracias a ello, fueron capaces de formular y recomendar posibles políticas exteriores y de defensa en función de estas.

En consideración de lo mencionado, en el presente artículo se realiza un repaso de las principales concepciones geopolíticas para identificar aquellos factores que hacen que un determinado espacio geográfico se vuelva parte del interés de un Estado. Luego, se lleva a cabo un estudio de caso holístico cualitativo (Bennett y Elman, 2007) que permite, por un lado, elaborar un resumen de los principales estudios que realizan un análisis geopolítico del Atlántico Sur Occidental a la luz de las principales concepciones geopolíticas identificadas y, por otro lado, analizar los documentos oficiales tanto del Ministerio de Defensa como del Ministerio de Relaciones Exteriores y Culto de la Argentina. El trabajo echa luz sobre dos cuestiones vinculadas a la geopolítica del Atlántico Sur: por un lado, muestra que las características del Atlántico Sur Occidental coinciden con aquellas que son identificadas - por las principales tradiciones geopolíticas-como necesarias para que un Estado le de relevancia a un determinado espacio geográfico, y por otro lado, permite evidenciar - a partir del análisis documental- que el Estado argentino caracteriza al Atlántico Sur Occidental como espacio geopolíticamente relevante para sus intereses.

\section{Los estudios geopolíticos entre la espacialidad y el interés nacional}

Gran parte de las tradiciones geopolíticas, desde su surgimiento hasta la actualidad, han vinculado implícitamente factores de análisis con el interés de los Estados. En el presente artículo, se define la geopolítica como "todo lo relacionado con las rivalidades por el poder o la influencia sobre determinados territorios y sus poblaciones: rivalidades entre poderes políticos de todo tipo (...) por el 
control o el dominio de territorios de mayor o menor extensión" (Lacoste, 2012, p.8). En este marco, se identifican tres tradiciones geopolíticas que se caracterizan por una concepción determinada de la espacialidad, el interés de los actores sobre ella y los elementos que contribuyen a determinar o construir el mismo.

En primer lugar, la escuela geopolítica clásica surgió a finales del siglo XIX y tuvo vigencia hasta la Segunda Guerra Mundial, en donde se realizaba un análisis material del escenario geopolítico y utilizaban metáforas organicistas para justificar la expansión geográfica del Estado. En este sentido, la escuela geopolítica alemana -surgida a finales del siglo XIX y principios del siglo XXha sido considerada como la pionera en lo que respecta al desarrollo de estudios de geopolítica clásica.

Los principales exponentes de esta escuela fueron Rudolf Kjellen, Friederich Ratzel y Karl Haushofer y, más allá de las distintas aproximaciones que hacen a los estudios de geopolítica, los tres identifican fuertes conexiones entre la geografía y el interés de los Estados. Kjellen (1985) vincula al bienestar ciudadano con la expansión del Estado; Ratzel (1975) relaciona al territorio controlado por un Estado y el crecimiento de su población con la conquista y, por último, Haushofer (1985) establece conexiones entre las reservas de territorio sin ocupar que posee un Estado y la "necesidad" de expandirse cuando ya no quedan territorios libres a ocupar por la propia población.

Otro autor relevante en las primeras décadas de desarrollo de los estudios geopolíticos fue Halford Mackinder (1919) quien, desde una mirada geopolítica vinculada a los intereses del Reino Unido, estableció los lineamientos que debería seguir su Estado para poder neutralizar cualquier amenaza que provenga de la entidad política que controle lo que él denomina el "heartland" de la región de Eurasia. Al igual que el caso de la escuela geopolítica alemana, Mackinder también establece una fuerte relación entre el interés del Estado y la geografía, en donde la conquista, control u proyección de poder hacia determinado territorio es necesario para alcanzar el interés del Estado.

En segundo lugar, destacamos a la geopolítica neoclásica como segunda tradición geopolítica relevante. La misma realiza un análisis material de los factores geopolíticos, pero, a diferencia de la escuela geopolítica clásica, no utilizan metáforas organicistas, sino que justifican la conducta del Estado -a partir motivaciones geopolíticas- considerando sus intereses de seguridad, económicos y de influencia. En otras palabras, no justifican la conducta del Estado a partir de "necesidades naturales" de expansión, sino que el comportamiento de este pasa a entenderse en función de sus intereses de seguridad, influencia y económico-comerciales. 
Hay varios exponentes reconocidos de la geopolítica neoclásica -surgida luego de la Segunda Guerra Mundial- (Dolman, 2012; Friedman, 2009; Kaplan, 2000; Huntington, 1993; Klare, 2003), sin embargo, en el presente artículo se consideran los aportes de Zbigniew Brzezinski, Henry Kissinger y George Kennan. Este recorte se justifica en el hecho de que estos autores tuvieron fuertes conexiones con el diseño de la política exterior de sus Estados luego o durante su producción académica, lo que refuerza la idea de que hay una conexión entre el análisis geopolítico y los intereses de un determinado Estado.

En primer lugar, Brzezinski logró articular, mediante el concepto "geoestrategia", la política exterior estadounidense con los intereses nacionales en función del momento unipolar y de las características geográficas de Eurasia. En palabras del autor

-Eurasia- es donde podría surgir, en un momento dado, un rival potencial de los Estados Unidos. Por lo tanto, el punto de partida para la formulación de la geoestrategia estadounidense para la gestión a largo plazo de los intereses geopolíticos estadounidenses en Eurasia debe centrarse en los jugadores claves y en una adecuada evaluación del terreno (Brzezinski, 1997, p. 48).

En segundo lugar, Kissinger (1994) en su cargo como Secretario de Estado de Nixon realizó un análisis geopolítico de la confrontación bipolar y, a partir del mismo, ideo su plan de acercamiento a los Estados Unidos de América a China para abrirle a la Unión Soviética otro frente de preocupación, en donde ya no solo tendría que mirar para el Oeste, sino también para el Este. En tercer lugar, Kennan (1947) fue el ideólogo de la doctrina de contención, en donde especificaba que la Unión Soviética colapsaría por las propias deficiencias de su sistema de producción, por lo que los Estados Unidos de América solo deberían limitarse a evitar que la Unión Soviética se expanda territorialmente. En este sentido, si el interés norteamericano versaba en ganar la confrontación bipolar, debía asegurarse de que los soviéticos no conquisten otros territorios.

Ahora bien, en tercer lugar, se encuentra a la geopolítica crítica como un enfoque que cuestiona la vigencia de aquellas tradiciones geopolíticas que buscan analizar e identificar los elementos que están presentes en un determinado espacio geográfico e influyen en el potencial interés estatal. De esta forma, autores como Ó Tuathail (1996) cuestionan la "imaginación geopolítica moderna" que pregonan ciertos enfoques para concentrarse en dar cuenta de la manera en la que se construye el discurso espacial de las políticas exteriores de los Estados (Coronado y Uc, 2010, p.65). 
Uno de los precursores de esta tradición fue Agnew (2005), quien cuestionó la espacialidad del poder establecido por los enfoques neoclásicos al sostener el argumento de que el control ejercido por el Estado no es igual en todos los lugares de su territorio. En este sentido, argumenta que en la actualidad el poder ejercido sobre un espacio determinado "comienza a ser aplicado por parte de gobiernos locales y regionales y de comunidades suprarregionales para fines tales como el desarrollo económico o la identidad política, sin que vaya acompañado del poder coercitivo tradicionalmente asociado a los Estados territoriales" (Agnew, 2005, p. 313-314).

En función de este argumento, el autor plantea que la imaginación geopolítica moderna implica la vigencia de una "trampa territorial" que supone que la soberanía de los Estados modernos requiere espacios claramente definidos territorialmente, que hay asuntos internos y externos claramente diferenciables $\mathrm{y}$, por último, que el Estado sirve de contención geográfico de su sociedad. De esta forma, la tradición geopolítica crítica comienza a cuestionar la idea de que los Estados detentan un poder absoluto sobre los espacios geográficos en pos de un objetivo claramente definido.

En función de lo mencionado, la conducta de un Estado en un determinado espacio geográfico se enfrenta con problemas vinculados a la "imaginación geopolítica moderna", en donde los Estados nacionales no tienen el control absoluto del territorio ni funcionan como contenedor geográfico de su ciudadanía. No obstante, los Estados siguen planeando y ejecutando su política exterior y de defensa sobre los espacios geográficos en función de determinados objetivos (Battaglino, 2010; Russell, 1990). De esta manera, como muestran los enfoques geopolíticos neoclásicos, los Estados definen sus políticas y objetivos que se proyectan en un determinado espacio geográfico a partir de la identificación de ciertos intereses, pudiendo estos estar vinculados con asuntos de seguridad (Friedman, 2009; Kaplan, 2000), de economía (Kennan, 1947; Klare, 2003) o simplemente una construcción histórica nacional relacionada con el apego a un determinado espacio geográfico que es considerado como propio (Escudé, 1988; 1992).

\section{El Atlántico Sur Occidental y sus principales características}

Los geopolíticos argentinos han demostrado durante todo el siglo XX un notable interés por la región del Atlántico Sur Occidental (Mapa 1), tanto en lo 
vinculado a la seguridad como en lo ligado a la economía (Assef, 1980; Guglialmelli, 1979; Hernández y Chitarroni, 1977; Storni, 1967).

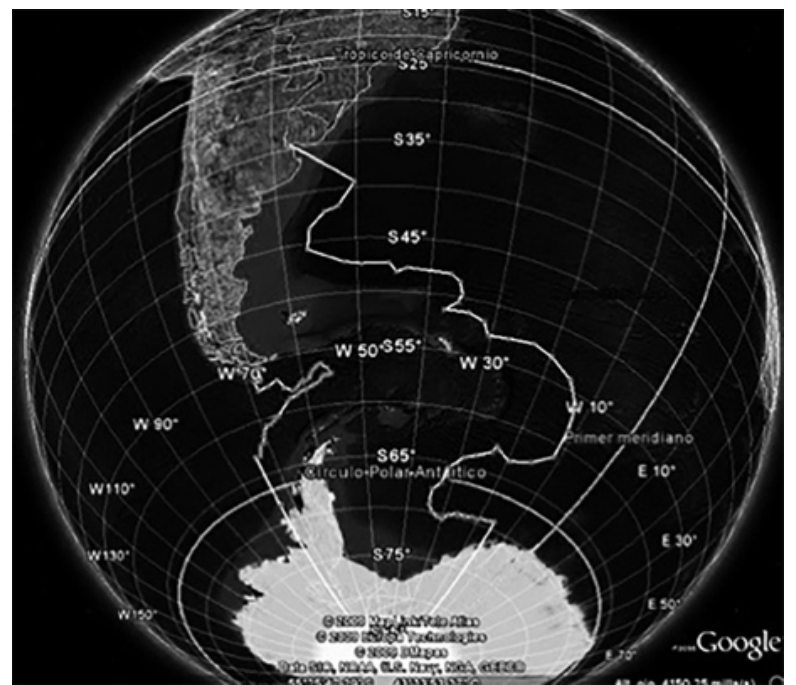

Figura 1. Mapa de Atlántico Sur

Fuente: Google Earth.

Ya iniciado el siglo XXI, los escritos argentinos sobre geopolítica del Atlántico Sur Occidental se alejaron de las visiones ligadas al nacionalismo territorial ${ }^{2}$ y se acercan a identificar aquellos elementos materiales que caracterizan dicha región y que pueden ser de interés para la Argentina. En esta línea, los autores afirman, por un lado, que esta zona en la actualidad es altamente estratégica (Eller y Quintana, 2017) y, por otro lado, que a medida que transcurra el tiempo, esta región va a ser cada vez más importante no solo para los Estados que la circundan (Bertotto, 2014), sino para el conjunto de los Estados del sistema internacional (Ayuso y Viilup, 2013).

Estas dos afirmaciones que sostienen la importancia estratégica del Atlántico Sur están basadas, a su vez, en dos hechos: en primer lugar, en la presencia de una gran cantidad de recursos naturales en la región (Koutoudjian, 2011) y, en segundo lugar, en la ubicación de ésta respecto a la Antártida (Altieri, 2017, p.62). Para entender a esta región en las dos dimensiones que la hacen relevante,

2 El Realismo Periférico hace una fuerte crítica al nacionalismo territorial, en donde dicho concepto refiere a la importancia de un territorio para un Estado basado en una estructura de significado producto de la propia construcción nacional de su historia y de cómo la misma fue transmitida de generación en generación mediante su sistema educativo. 
es necesario pensar al Atlántico Sur como un sistema compuesto por tres zonas que están estrechamente vinculadas entre sí. En primer lugar, (1) las Islas Malvinas; en segundo lugar, (2) el Arco de las Antillas Australes, y en tercer lugar (3) la Antártida.

Sobre las Islas Malvinas (1) hay que tener en cuenta dos factores: los recursos naturales allí presentes y la posición geográfica de las islas. En relación con los recursos naturales, éstos pueden identificarse en dos grupos, los energéticos y los ictícolas. El primer grupo se concentra en dos cuencas que actualmente están dentro de un área cuya soberanía es disputada entre Gran Bretaña y la Argentina, la Cuenca Malvinas Norte y la Cuenca Malvinas Sur (Archibaldo Lanús, 2016 p. 429).

Actualmente, es en la cuenca norte en donde se ha recabado la información necesaria que permite proyectar la explotación de gas y petróleo hallado (principalmente por el descubrimiento geológico 'Sea Lion') (Magnani, 2019). Con respecto a la cuenca sur, en 2012 se constató la presencia de gas condensado (Archibaldo Lanús, 2016, p.467). Además, ésta presenta un desafío tecnológico ya que es más profunda que la cuenca norte y se encuentra en el borde del Talud Continental (Koutoudjian, 2011, p.37).

El segundo grupo de recursos, provenientes del mar, existen en abundancia ya que conforman gran parte del porcentaje de lo que se exporta desde las Islas Malvinas. En números, $89 \%$ de lo que las Islas exportan son recursos provenientes del mar $(77 \%$ de Moluscos, $15 \%$ de pescado congelado sin filete y $1,9 \%$ de filete de pescado) contra el $11 \%$ de otros productos (entre los que se destacan el $6,4 \%$ de lana y el 1,5\% de carne de oveja y cabra) (Observatory of Economic Complexity, 2016). En síntesis, los recursos vivos son el corazón económico de las Islas Malvinas, cuyas áreas de pesca establecidas en las licencias a barcos pesqueros otorgados por el gobierno de las Islas son eje de conflicto con la Argentina (Koutoudjian, 2011, p.75). Mientras que los recursos energéticos constituyen la principal riqueza económica potencial que pueden tener las islas en los años venideros.

Si tenemos en cuenta la posición geográfica de las Malvinas, con ver el mapa nos podemos percatar de su importancia. Si bien el control de las Islas no es utilizado por Gran Bretaña como argumento para sustentar su reclamo de soberanía en la Antártida (Eissa y Caplan, 2015), el mismo puede ser utilizado como punto logístico operativo para planear y ejecutar sus actividades antárticas, proyectar su poder militar hacia el continente blanco y hacia los tres pasajes interoceánicos (Cabo de Hornos, Canal de Beagle y el Estrecho de Magallanes) 
(Magnani, 2019, p.148). Si bien estos pasajes se encuentran lejos de las principales vías de navegación mundial, el pasaje por el Cabo de Hornos

constituye una de las dos alternativas al transporte de sustancias peligrosas entre Asia y Europa o Norteamérica, las que tienen vedadas el paso por pasajes interoceánicos como el Canal de Panamá o Suez y solo pueden circular por aguas abiertas durante su tránsito (Koutoudjian, 2011, p. 54).

Por último, cabe destacar que la Argentina no requiere de las Islas Malvinas para realizar sus actividades en el continente blanco, proyectarse hacia él y mantener el control de los mencionados pasajes bioceánicos (Child, 1990). Sin embargo, "si el país pudiera negárselas a Gran Bretaña, reforzaría considerablemente su reclamo antártico" (Child, 1990, p.195) y el control de la zona que más amenazas plantea a su seguridad.

En segundo lugar, (2) el Arco de las Antillas Australes está compuesto por las islas Georgias del Sur, Sandwich del Sur, Orcadas del Sur y Shetland del Sur. Las dos primeras están bajo el control militar de Gran Bretaña, mientras que las últimas dos se encuentran bajo la jurisdicción del Tratado Antártico al estar al sur del paralelo $60^{\circ} \mathrm{S}$. En este sentido, es necesario remarcar que estas son importantes ya que "si las bases logísticas de [una] nación se encuentran distantes, como en el caso de Gran Bretaña, las islas del Arco de Scotia desempeñan un papel importante como zona de apoyo y escala para las actividades antárticas" (Child, 1990, p.195).

Además, en lo que hace a la disputa por la soberanía de las islas Sandwich y Georgias del Sur entre la Argentina y Gran Bretaña, hay un par de cosas que hay que tener en cuenta. Por un lado, que "estos archipiélagos no poseen población permanente, por lo cual no vemos motivo alguno para que el Reino Unido justifique sus pretensiones sobre este territorio en base al Derecho de Autodeterminación de los Pueblos" (Eissa y Caplan, 2015, p.10). Por otro lado, que la conformación geológica de las Antillas Australes indica que "la zona antártica podría estar ligada a estos archipiélagos por la prolongación de la Cordillera de Los Andes que continúa hasta la Península Antártica pasando también por las Orcadas y las Shetland del Sur" (Eissa y Caplan, 2015, p.11).

Las Malvinas quedan fuera de esta conformación geológica ya que son una extensión de la plataforma continental y no de la prolongación cordillerana. Estas dos peculiaridades mencionadas que diferencian a estas islas con las Malvinas son importantes para la Argentina a la hora de formular argumentos basados en el derecho internacional orientados a resolver la disputa con Gran Bretaña. 
Un aspecto jurídico de gran relevancia que afecta a gran parte del Atlántico Sur (del paralelo $60^{\circ} \mathrm{S}$ al Ecuador entre el meridiano $20^{\circ} \mathrm{O}$ y el meridiano de Cabo de Hornos) y que incluye a las Islas Malvinas y a las Antillas Australes es el Tratado para la Proscripción de Armas Nucleares de América Latina y el Caribe que entró en vigor en 1969, y del cual 33 países latinoamericanos son actualmente signatarios. El tratado indica que

1- Las Partes Contratantes se comprometen a utilizar exclusivamente con fines pacíficos el material y las instalaciones nucleares sometidos a su jurisdicción, y a prohibir e impedir en sus respectivos territorios: a. El ensayo, uso, fabricación, producción o adquisición, por cualquier medio, de toda arma nuclear, por sí mismas, directa o indirectamente, por mandato de terceros o en cualquier otra forma, y b. El recibo, almacenamiento, instalación, emplazamiento o cualquier forma de posesión de toda arma nuclear, directa o indirectamente, por sí mismas, por mandato a terceros o de cualquier otro modo.

2- Las Partes Contratantes se comprometen, asimismo, a abstenerse de realizar, fomentar o autorizar, directa o indirectamente, el ensayo, el uso, la fabricación, la producción, la posesión o el dominio de toda arma nuclear o de participar en ello de cualquier manera (Artículo I del Tratado de Tlatelolco, 1969).

Si bien Gran Bretaña no es una parte contratante del tratado, es interesante ver que el espíritu fundamental del tratado es proscribir en la región de América Latina $^{3}$-incluido el Atlántico Sur-el uso de armas nucleares, haciendo que esta zona sea considerada libre de armas nucleares independientemente del hecho de que no todos los Estados que tienen presencia en la región son signatarios de este.

3 El Tratado del Tlatelolco explicita el área de aplicación del mismo en su artículo IV, considerando al espacio geográfico situado "en el hemisferio Occidental dentro de los siguientes límites (excepto la parte del territorio continental y aguas territoriales de los Estados Unidos de América): comenzando en un punto situado a $35^{\circ}$ latitud norte y $75^{\circ}$ longitud oeste; desde allí directamente al sur hasta un punto a $30^{\circ}$ latitud norte y $75^{\circ}$ longitud oeste; desde allí directamente al este hasta un punto a $30^{\circ}$ latitud norte y $50^{\circ}$ longitud oeste; desde allí por una línea loxodrómica hasta un punto a $5^{\circ}$ latitud norte y $20^{\circ}$ longitud oeste; desde allí directamente al sur hasta un punto a $60^{\circ}$ latitud sur y $20^{\circ}$ longitud oeste; desde allí directamente al oeste hasta un punto a $60^{\circ}$ latitud sur y $115^{\circ}$ longitud oeste; desde allí directamente al norte hasta un punto a $0^{\circ}$ latitud y $115^{\circ}$ longitud oeste; desde allí por una línea loxodrómica hasta un punto a $35^{\circ}$ latitud norte y $150^{\circ}$ longitud oeste; desde allí directamente al este hasta un punto a $35^{\circ}$ latitud norte y $75^{\circ}$ longitud oeste" (Artículo IV Tratado de Tlatelolco, 1969). 


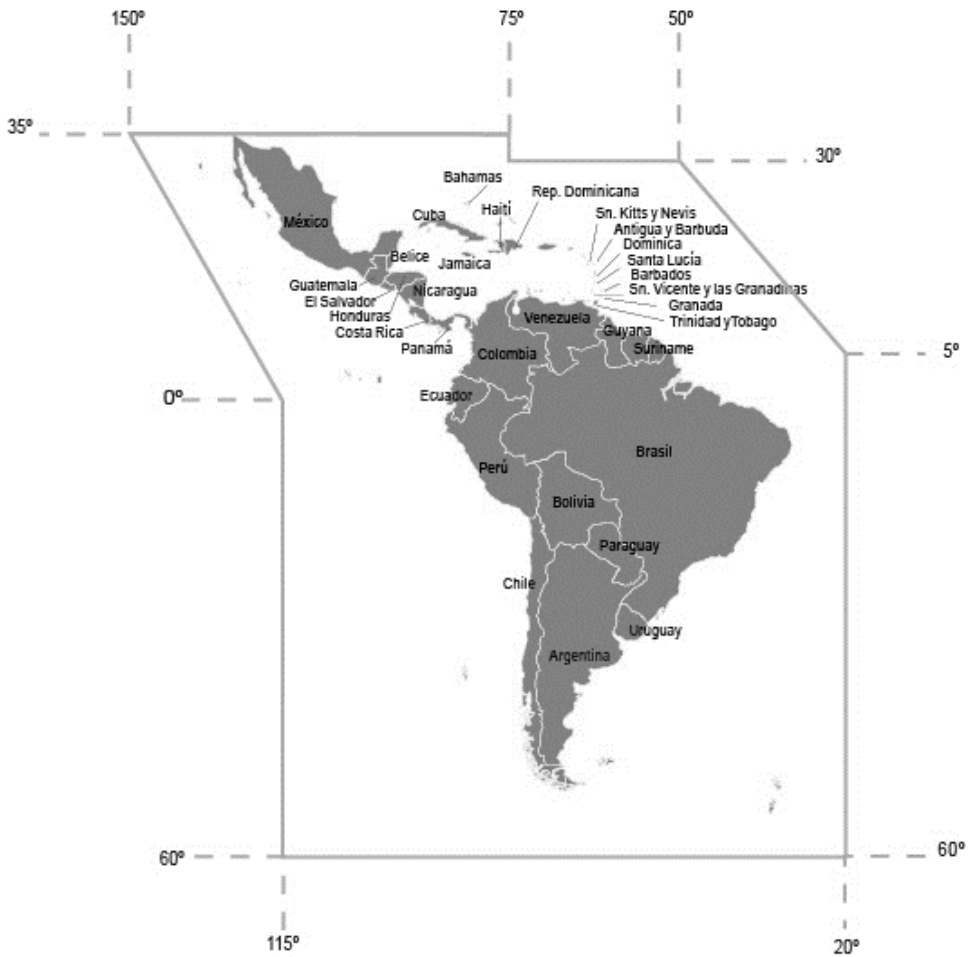

Figura 2. Área de aplicación del Tratado de Tlatelolco Fuente: OPANAL.

En tercer lugar, (3) la Antártida es una zona que, a pesar de que no es estrictamente parte del Atlántico Sur, no puede dejar de considerarse en los análisis geopolíticos de la región ya que ésta es el principal punto de acceso al continente blanco. Para tener dimensión de la relevancia estratégica de la Antártida es necesario comprenderla en sus dos características principales: Los recursos naturales que posee y su situación jurídica.

En términos de características geográficas, se puede decir que el continente blanco tiene una superficie de más de $14.000 .000 \mathrm{~km} 2$, una altura media de 2.000 metros sobre el nivel del mar, precipitaciones anuales que llegan a $150 \mathrm{~mm}$, temperaturas que llegan a $\operatorname{los}-90^{\circ} \mathrm{C}$ y que su Océano Antártico está rodeado por los Océanos Atlántico, Pacífico e Indico (Colacarai, 1998). Además, los otros continentes se encuentran considerablemente lejos de la Antártida, estando Sudamérica a $1.000 \mathrm{~km}$, seguida por Nueva Zelanda a $2.200 \mathrm{~km}$, Tasmania a $2.250 \mathrm{~km}$ y Sudáfrica a $3.600 \mathrm{~km}$. Es posible afirmar que su lejanía, sumado a su difícil acceso y sus condiciones climáticas extremas hacen que solo 
los Estados que están cerca o tienen las capacidades tecnológicas para llegar y operar en la zona puedan participar y sacar provecho del continente blanco.

A la hora de hablar de sus recursos y potencial económico, Colacarai (1998) menciona que "las potencialidades económicas que encierra han operado como un disparador del interés por parte de quienes lo consideran 'una fuente inagotable de recursos"” (1998, p.27). En sentido estricto, la Antártida cuenta con potencialidades económicas basadas en su enorme fuente de agua dulce, los recursos vivos, minerales y energéticos (Sepúlveda Cox, 2008).

Con respecto a los recursos vivos, se pueden destacar la posible -y clásica- explotación de focas y ballenas, la presencia de algas (importantes para el consumo animal, la industria textil y la fabricación de productos medicinales), de krill (Krill Fishery Report, 2018) y, en representación de la fauna ictícola, la existencia de alrededor de 120 especies de peces (Fraga, 1992).

Ahora bien, es imposible comprender la realidad de la Antártida sin ver el marco normativo que recae sobre ella y establece los parámetros de la conducta de los Estados que tienen presencia en dicho continente (Magnani, 2019, p. 150).

En este sentido, “el Tratado Antártico es la piedra angular del plexo normativo que afecta a la región antártica. El mismo -que se firmó el primero de diciembre de 1959 y está en vigencia desde el 23 de junio de 1962-" (Magnani, 2019, p. 150) puede ser visto como "un entramado de principios y objetivos a partir del cual comenzó a edificarse un sistema jurídico-político para administrar la cooperación internacional y la ciencia en la región" (Colacarai, 1998, p.94). A su vez, las ideas fuerza de este son tres: "(a) compromiso con la cooperación científica; (b) equilibrio pacífico y no militarización de la región; (c) desnuclearización" (Colacarai, 1998, p.96; Sampaio, 2015).

Con respecto los Estados miembros vinculados a la región antártica, es indicado mencionar que "el Tratado fue firmado originalmente por $12 \operatorname{Estados}^{4} \mathrm{y}$ fue adoptando más países signatarios ${ }^{5}$ en calidad de miembros consultivos y adherentes" (Magnani, 2019, p. 150-151). A su vez, a pesar de que previo a la constitución del Tratado existían reclamos de soberanía por parte de algunos Estados, “el art. IV del Tratado Antártico (...) soslayó los problemas de soberanía, pero a

4 Argentina, Australia, Bélgica, Chile, Francia, Japón, Nueva Zelanda, Noruega, Sudáfrica, URSS (Rusia asumió sus derechos en 1992 a causa de la desintegración de la Unión Soviética), Gran Bretaña y Estados Unidos.

5 El Tratado Antártico tiene 53 Estados signatarios en la actualidad. 
su vez mantuvo la posición de los Estados reclamantes, para no afectarlos en sus derechos" (Colacarai, 1998, p.100; Urbina Paredes, 2009; Lo Giudice, 2012).

Luego de la firma del Tratado, se incorporaron a éste -dando lugar a la creación del Sistema Antártico- dos convenciones y un protocolo. Las dos primeras son "La Convención para la Conservación de Focas Antárticas" y la "Convención para la Conservación de los Recursos Vivos Marinos Antárticos" " El segundo es el "Protocolo al Tratado Antártico sobre la Protección del Medio Ambiente" (Protocolo de Madrid), firmado en 1991 y vigente desde 1998 (Magnani, 2019, p. 151).

En este marco, "si bien todos los componentes del Sistema Antártico son fundamentales para la sólida cooperación internacional en la región, el Protocolo de Madrid $^{8}$ es cualitativamente relevante" (Magnani, 2019, p. 151). Esto se debe al hecho de que es fundamental para "la protección integral del ecosistema antártico y sus ecosistemas dependientes y asociados" (Colacarai, 1998, p. 129) en la medida que confirma la realización de inspecciones para verificar el cumplimiento de las normas ambientales acordadas y crea un Comité para la protección medioambiental de los ecosistemas de la zona (Roura, 2013). Además, a diferencia de las dos convenciones mencionadas, autores como Langone (1997), Laws (1989) acuerdan que, al establecer determinadas formas de proceder comunes a todos los Estados firmantes, el Protocolo de Madrid "denota su indisoluble vinculación y complementariedad con el Tratado Antártico" (Colacrai, 1998, p.130).

Sin embargo, como establece Moneta (1998), algo que no puede dejarse de lado es el hecho de que el Protocolo será abierto para su revisión en 2048, estando dentro de las posibilidades que el compromiso de los Estados firmantes con el ecosistema de la región no se renueve. De esta forma, la no ratificación del Protocolo daría una ventana de oportunidad a las acciones unilaterales de aquellos Estados que estén interesados en explotar los recursos de la zona (Magnani, 2019).

\section{Importancia otorgada por la Argentina al de Atlántico Sur Occidental}

En sintonía con lo desarrollado, en esta sección se analiza la relevancia geopolítica que tiene el Atlántico Sur Occidental para el interés argentino a la luz de las

6 Firmada en 1972 y en vigencia desde 1978.

7 Firmada en 1980 y puesta en vigencia en 1982.

8 Firmado hasta la actualidad por 37 Estados. 
características de dicha región, tanto en lo que respecta a la importancia para la seguridad como en lo que hace a su potencial económico. En esta línea, el Libro Blanco de la Defensa Argentina en su capítulo III indica que

La ratificación de la legítima e imprescriptible soberanía sobre las Islas Malvinas, Georgias y Sandwich del Sur y los espacios marítimos e insulares correspondientes por ser parte integrante del territorio nacional y el afianzamiento de los derechos argentinos de soberanía sobre el Sector Antártico Argentino, son cuestiones centrales de la política exterior de la nación. (2015, p. 40).

Con respecto al factor económico, como ya se mencionó, los abundantes recursos naturales presentes en el Atlántico Sur y la cercanía que tiene la Argentina con dicha región hacen que no sea impensable considerar que el país participe en la explotación de estos. Por lo tanto, una de las razones por la cual la Argentina considera al Atlántico Sur Occidental como un espacio geopolíticamente relevante está dada por la alta probabilidad que tiene el Estado argentino de participar -en un futuro- de la extracción, consumo y comercialización de los recursos naturales de la zona. En este sentido, el Libro Blanco de la Defensa Argentina establece la preocupación del país por "la ilegal concesión de licencias de pesca por parte del Reino Unido que pone en riesgo la sustentabilidad de la explotación de los recursos ictícolas" (Libro Blanco de la defensa argentina [LBDA], 2015, p. 41).

Por otro lado, con respecto al factor ligado a la seguridad, es necesario destacar que, si bien en la actualidad la zona del Atlántico Sur es pacífica y no plantea inconvenientes ni preocupaciones inmediatas para el país, las características propias de dicha región hacen que haya probabilidades de que en un futuro la seguridad de la Argentina se vea comprometida. En referencia a esto, la Directiva Política de Defensa Nacional (DPDN) (2014) en su sección de Apreciación Estratégica del Escenario Regional marca tres cuestiones vinculadas a la probabilidad de escenarios conflictivos en un futuro y el posicionamiento que la Argentina debe tener para defender sus intereses.

- En primer lugar, establece "la comprobación de que la región suramericana cuenta con una dotación de recursos energéticos y naturales no renovables comparativamente superior a la existente en otras regiones del mundo" sumado a "la verificación de los yacimientos de petróleo y gas no convencional ubicados en la Patagonia argentina que constituyen la tercera reserva mundial de este tipo de recurso hidrocarburífero". 
- En segundo lugar, que la presencia de estos recursos estratégicos en la región cobra relevancia "en un contexto caracterizado por la tendencia internacional hacia una creciente competencia entre los Estados-Nación por el acceso y control de los recursos naturales estratégicos, entre ellos, petróleo, gas, minerales, agua dulce y alimentos".

- Por último, en tercer lugar, que la "la estructuración del Sistema de Defensa de la República Argentina (...) deberá contemplar esta tendencia del escenario internacional en sus previsiones estratégicas" (DPDN, 2014, p. 8).

A su vez, documentos oficiales del Ministerio de Defensa también identifican a la presencia y conducta británica en el Atlántico Sur como un problema para la seguridad de la Argentina. Por un lado, se destaca la preocupación por las actividades unilaterales del Reino Unido debido a la exploración de hidrocarburos que viene teniendo lugar en dicha región gracias al aval británico. De esta forma, se menciona que

los actos unilaterales británicos entrañan un riesgo inconmensurable para la defensa de la región sudamericana, [identificando como un riesgo potencial] la posibilidad de que como resultado de la falta de medidas adecuadas (...) por parte de las empresas británicas que realizan ilegítimas actividades de exploración y eventual explotación de hidrocarburos en la plataforma continental argentina se produzcan graves daños ecológicos (LBDN, 2015, p. 41).

Por otro lado, se manifiesta la "preocupación por el despliegue militar británico en el Atlántico Sur y la realización de ejercicios militares" (LBDN, 2015, p. 43), en donde se destaca la posibilidad de que el Reino Unido ingrese armas nucleares a una región que ha sido declarada por los países sudamericanos como una zona de paz, de cooperación y donde rige la prohibición a la introducción de armas de destrucción masiva.

Ahora bien, esta preocupación por la depredación de los recursos naturales junto con los asuntos vinculados a la seguridad está estrechamente vinculados con el posicionamiento geopolítico de la Argentina. Un factor altamente condicionante para la Argentina son sus $4.725 \mathrm{~km}$ de costa (litoral atlántico) que, teniendo en cuenta el área de zona económica exclusiva (ZEE), implican una superficie marítima de $1.530 .000 \mathrm{~km} 2$ de la cual el Estado se tiene que hacer cargo. Esta característica geográfica tiene al menos tres implicancias. Por un lado, la primera es la realidad de que

por el mar el país conduce el $90 \%$ de su comercio exterior, (...) es por ello que es sensible a sus vías de comunicación marítima y necesita controlarlas 
en la paz y mantenerlas expeditas en caso de un conflicto armado para asegurar el bienestar y progreso de su pueblo (Koutoudjian, 2011, p. 77).

Por otro lado, hay una inconmensurable fuente de recursos y riqueza que puede proporcionar el mar argentino. La segunda implicancia es que actualmente hay vastas zonas de la ZEE que contienen importantes yacimientos de hidrocarburos que actualmente no están siendo explotados (Tabla 1). Estos yacimientos, sumado a las cuencas que actualmente están siendo explotadas y a los enormes yacimientos continentales que también están sin producción, dan a la Argentina un importante potencial en términos de producción, consumo y exportación de energía.

Tabla 1

Cuencas de hidrocarburos con y sin producción dentro del territorio argentino.

\begin{tabular}{|l|c|c|}
\hline \multicolumn{1}{|c|}{ Cuenca } & Petróleo (miles de $\mathbf{~ m}^{\mathbf{3}}$ ) & Gas (millones de $\mathbf{~ m}^{\mathbf{3}}$ ) \\
\hline Austral & 13559 & 110653 \\
\hline Cuyana & 22480 & 744 \\
\hline Golfo de San Jorge & 251163 & 47849 \\
\hline Neuquina & 78604 & 138960 \\
\hline Noroeste & 4568 & 30052 \\
\hline
\end{tabular}

Fuente: Libro blanco de la defensa argentina (2015).

Finalmente, la tercera implicancia está relacionada con los recursos vivos presentes en la ZEE del país. Los sistemas marinos y costeros del litoral atlántico "se encuentran entre los más productivos del mundo, sustentan una compleja interacción de ecosistemas y contienen una enorme biodiversidad. Allí se encuentran especies de alto valor nutritivo y comercial" (Koutoudjian, 2011, p.52). Esto conlleva que el país tiene la posibilidad de convertirse en un futuro, si lo desea e invierte lo necesario, en un gran exportador de alimentos provenientes de la pesca.

En función de las presentes implicancias producto del posicionamiento geopolítico del país, pueden verse -en los documentos del Ministerio de Relaciones Exteriores y Culto y del Ministerio de Defensa- el interés de la Argentina por consolidar su presencia en el Atlántico Sur Occidental, fortaleciendo su reclamo soberano en la arena internacional y estableciendo su voluntad de realizar un control material sobre aquella región.

La creación de la Comisión Nacional del Límite de la Plataforma Continental (COPLA) en 1997 para que sea el órgano estatal encargado de elaborar la presentación final del límite exterior de la plataforma continental argentina -de conformidad con la CONVEMAR y la Ley N²3.968 de Espacios Marítimos-, 
es un indicador de los intereses del Estado argentino en el Atlántico Sur Occidental. Con dicha presentación realizada el 21 de abril de 2009 y aprobada el 11 de marzo de 2016, la Argentina logró aumentar unos 1782500 km² de territorio soberano en la plataforma continental -equivalente al $48 \%$ del territorio emergido-a partir de una ampliación del límite de su territorio a 350 millas naúticas de la costa.

La labor de este organismo estatal pone en evidencia una política de Estado que la República Argentina viene desarrollando coherente e ininterrumpidamente desde hace 20 años, reafirmando sus intereses sobre los archipiélagos del Atlántico Sur y la Antártida Argentina. En los documentos de la comisón se destaca que

La Argentina ha considerado las tareas del trazado de su límite más extenso como una política de Estado y ha mantenido una continuidad del equipo de trabajo desde 1997. COPLA ha desarrollado sus tareas con profesionales contratados expresamente para el trabajo y con la colaboración de los organismos del Estado vinculados a la temática (Límite Exterior de la Plataforma Continental Argentina, 2016, p.2)

Dentro de este objetivo de política exterior delineado, el Ministerio de Defensa identifica en la DPDN las responsabilidades de la Defensa Nacional que, "bajo la dirección del Ministerio de Relaciones Exteriores y Culto, apoya materialmente las acciones tendientes a fundamentar los reclamos soberanos de referencia y hacer operativos los objetivos políticos concretos que de ello se deriven" (DPDN, 2014, p. 9). De esta forma, este pasaje hace explícito que la política de defensa se complementa con la política exterior para contribuir a la materialización del interés identificado por la Argentina.

En función de las implicancias que tiene para la Argentina sus extensas costas y el reconocimiento de este posicionamiento geopolítico del país por parte de los documentos oficiales analizados, puede inferirse que cualquier escenario extraordinario que se dé en el Atlántico Sur tiene repercusiones inmediatas en lo que hace a la seguridad de la Argentina. En consiguiente, es posible identificar dos tipos de escenarios que pueden llegar a ocurrir en las próximas décadas en el Atlántico Sur, uno de índole económico y otro de índole político-militar.

El escenario económico puede abarcar un amplio espectro de situaciones e incluso ser el puntapié inicial del escenario político-militar. Dentro de las principales situaciones posibles podemos destacar dos. En primer lugar, un accidente relacionado con la extracción de petróleo en alguna de las cuencas que están bajo el control británico, lo que podría afectar -por su impacto ambiental- a la población 
argentina de la costa patagónica, la actividad pesquera y cualquier proyecto de extracción de hidrocarburos de cuencas submarinas dentro de la ZEE.

En segundo lugar, también puede darse en las próximas décadas un escenario en donde los principales Estados comiencen a cuestionar el consenso en materia de cooperación que regula la dinámica estatal en la Antártida desde 1961, haciendo que el Sistema Antártico (especialmente el Protocolo de Madrid) sea revisado. En consecuencia, la cooperación en la región se vería fuertemente afectada provocando que los Estados perciban que las acciones unilaterales le traerán mayores beneficios al corto plazo que lo que le traerán las acciones multilaterales al largo plazo. En efecto, la discordia aumentaría y habría un creciente conflicto sobre quiénes pueden explotar qué y dónde.

Con respecto al escenario político-militar, si este tiene lugar, lo más probable es que sea una consecuencia de la merma en la cooperación y el aumento del conflicto entre Estados por la explotación económica de los recursos, principalmente, de la Antártida. En sentido estricto, el escenario político militar remite a la conflagración entre dos o más Estados y el consecuente incremento de los actores beligerantes debido al carácter internacional -ausencia de soberanía- de la región antártica. Si este escenario tiene lugar, el Atlántico Sur sería inmediatamente considerado un escenario en disputa, en donde las Islas Malvinas, las Antillas Australes y la Patagonia serían de alto interés estratégico por sus características geográficas que habilitan la proyección de poder propio y de los aliados hacia la zona del conflicto y la denegación de acceso de los enemigos a dicha zona.

A su vez, el estallido de una conflagración a pocos kilómetros de su territorio afectaría el desempeño económico de la Argentina en la medida que cualquier actividad económica ligada al comercio marítimo y a la actividad extractiva en la ZEE se vería cuestionada. Además, el mismo conflicto bélico a pocos kilómetros del país sería un riesgo y una amenaza para la Argentina. En pocas palabras, cualquier confrontación estatal en el Atlántico Sur pondría en jaque a la seguridad de los ciudadanos y habitantes argentinos.

\section{Reflexiones finales}

El presente artículo analizó la región del Atlántico Sur Occidental a la luz de las principales tradiciones geopolíticas. En este marco, si bien se coincide con la geopolítica crítica al entender que dar por sentado el dominio absoluto de un Estado sobre un territorio es un error, se retoman a los escritos de la geopolítica neoclásica ya que se entiende que una determinada región puede poseer 
elementos vinculados al campo económico y de seguridad que influyen en el interés de un Estado, especialmente si el mismo tiene un posicionamiento geopolítico que lo vincula inexorablemente con dicha región.

En este sentido, el Atlántico Sur Occidental -debido a su potencial económico, a las disputas de soberanía sobre sus archipiélagos y a su cercanía con la Antártida- posee características que son potencialmente atractivas para los Estados que son parte de dicho escenario o que pueden estar presentes en la misma gracias a sus capacidades materiales, logísticas y tecnológicas.

De esta forma, se identifica a partir del análisis documental que la Argentina posee un claro interés en el Atlántico Sur Occidental. Los documentos oficiales del Ministerio de Defensa muestran una preocupación del Estado argentino tanto por la depredación de los recursos naturales a causa de las licencias pesqueras que otorga el gobierno de las Islas Malvinas como por los ejercicios militares que realiza el Reino Unido en dicha región (sumado a la potencial presencia de sistemas de armas nucleares).

En esta línea, el presente artículo muestra que existen sobre el Atlántico Sur Occidental intereses argentinos ligados a la seguridad y a la economía que van más allá de reivindicaciones nacionales históricas y se vinculan tanto con la voluntad de la Argentina de controlar los recursos de dicha región como con la percepción de amenaza que implica la presencia del Reino Unido en ella.

\section{Referencias}

Agnew, J. (2005). Geopolítica: una revisión de la política mundial. Madrid: Trama Editorial.

Altieri, M. (2017). Poder Naval en el Atlántico Sur Occidental del SXXI. Nuevo escenario mundial y competencia por el control del espacio marítimo entre los años 2002 y 2015, Tesis de Maestría. Buenos Aires; Argentina: Universidad de la Defensa, Escuela Superior de Guerra “Tte. Grl. Luis María Campos”.

Archibaldo Lanús; A. (2016). Repensando Malvinas. Avellaneda: El Ateneo.

Assef, A. (1980). Proyección continental de la Argentina. Buenos Aires: Pleamar.

Ayuso, A y Viilup, E. (2013). Una nueva mirada al Atlántico. CIDOB d'afers Internacionals, 102 (103), 7-27.

Battaglino, J. (2010). "La política militar de Alfonsín: la implementación del control civil en un contexto desfavorable". En Gargarella,R.;Murillo, M. y Pecheny, M. (Eds.), Discutir Alfonsin (pp. 161-184). Buenos Aires: Siglo XXI. 
Bennett, A. y Elman, C. (2007) Case Study Methods in the International Relations Subfield. Comparative Political Studies, 40 (2): 170-195.

Bertotto, J. (2014). La isla de los Estados (RA) un lugar para el control de navegación (aérea y marítima) del Mar Argentino, que permitiría el ejercicio responsable de la soberanía nacional (tesis doctoral). Universidad del Salvador, Argentina.

Caplan, S. y Eissa, S. (2015). Análisis estratégico del Sistema Malvinas, Antártida y Atlántico Sur. Documentos de Trabajo No 28. Buenos Aires: Escuela de Defensa Nacional.

Colacarai, M. (1998). El Ártico y la Antártida: Su rol en las relaciones internacionales. Su relevancia desde la perspectiva ambiental. Rosario: CERIR y CECAR.

Comisión de Límites de la Plataforma Continental. (2016). Límite Exterior de la Plataforma Continental Argentina.

Comisión para la Conservación de los Recursos Vivos Marinos Antárticos. (2018). Krill Fishery Report.

Child, J. (1990). “Geopolítica sudamericana y la Antártida ¿confrontación o cooperación?”. En Kelly, P. y Child, J. (Eds.), Geopolítica del Cono Sur y la Antártida (pp. 191-207). Buenos Aires: Pleamar.

Dolman, E. (2012) New Frontiers, Old realities. Strategic Studies Quarterly, 6 (1), $78-96$.

Eissa, S. (2013). Política exterior y política de defensa en argentina: dos caras de la misma moneda. Perspectivas de Politicas Públicas, 3(5), 171-191.

Eller, M., y Quintana, P. (2017). "La importancia Geopolítica del Atlántico Sur. A 50 años de la Resolución 2065”. En Bologna, B. (Ed), Malvinas y la construcción del reclamo soberano, pasado, presente y futuro (pp. 113-138). Buenos Aires: Universidad de La Plata.

Escudé, C. (1988). La declinación argentina. Argentina: Editorial de Belgrano.

Escudé, C. (1992). Realismo periférico: fundamentos para la nueva politica exterior argentina. Buenos Aires: Planeta Política y Sociedad.

Fraga, J. A. (1992). La Antártida, Reserva Ecológica. Buenos Aires: Instituto de Publicaciones Navales.

Friedman, G. (2009). The Next 100 Years. A forecast for the $21^{\text {st }}$ Century. New York: Doubleday.

Guglialmelli, J. (1979). Geopolítica del Cono Sur. Argentina: El cid editor. 
Haushofer, K. (1985). “Poder y Espacio”. En Rattenbach, A. (Ed), Antología Geopolitica (pp. 85-95). Buenos Aires: Editorial Pleamar.

Hernandez, J y Chitarroni, H. (1977). Malvinas, clave geopolítica. Buenos Aires: Castañeda.

Huntington, S. (1993). The clash of civilizations? Foreign Affairs, 72 (3), 3-27.

Kaplan, R. (2000). La anarquía que viene: la destrucción de los sueños de la posguerra fría. Barcelona: Ediciones B.

Kennan, G. (1947). The Sources of Soviet Conduct. Foreign Affairs, 25(4), 566-582.

Kissinger, H. (1994). La Diplomacia. México: Fondo de Cultura Económica.

Kjellen, R. (1985). “Autarquía”. En Rattenbach, A. (Ed), Antología Geopolítica (pp. 55-62). Buenos Aires: Editorial Pleamar.

Klare, M. (2003). Guerras por los Recursos. Barcelona: Urano.

Koutoudjian, A. (2011). Lineamientos para la incorporación de la problemática del mar argentino en la planificación territorial. Buenos Aires: Subsecretaria de planificación territorial de la inversión pública.

Lacoste, Y. (2012). Geopolítica: La larga historia del presente. Madrid: Síntesis Editorial.

Langone, J. (1977). Life at the bottom: The people of Antartica. Boston: Little, Brown \& Co.

Laws, R. (1989). Antartica. The last frontier. Londres: Cristopher Stocks.

Lo Giudice, J. I. (2012). La Antártida: pasado, presente y futuro desde la perspectiva argentina. VI Congreso de Relaciones Internacionales, Universidad Nacional de La Plata, Argentina.

Mackinder, H. (1919). Democratic ideals and reality. A study in the politics of reconstruction. New York: Holt \& Co.

Magnani, E. (2019) Los Estados Periféricos frente a sus intereses geopolíticos: el caso de la Argentina con el Atlántico Sur. Revista POSTData, 24(1): 139-176.

Ministerio de Defensa (2015). Libro Blanco de la Defensa Nacional. Buenos Aires: Ministerio de Defensa.

Ministerio de Defensa. (2014). Directiva Politica de Defensa Nacional.

Moneta, C. (1988). La Antártida en el sistema internacional del futuro. Buenos Aires: Grupo Editor Latinoamericano.

Ó Tuathail, G. (1996). The patterned mess of history and the writing of critical geopolitics: a reply to Dalby. Political Geography, 15 (6/7): 661-665. 
Organismo para la Proscripción de las Armas Nucleares en la América Latina y el Caribe (OPANAL). (2019). Zona de aplicación del Tratado. Recuperado de http://opanal.org/zona-de-aplicacion/.

Preciado Coronado, J. y Uc, P. (2010). La construcción de una geopolítica crítica desde América Latina y el Caribe. Hacia una agenda de investigación regional. Geopolítica(s), 1 (1): 65-94.

Protocolo al Tratado Antártico sobre Protección del Medio Ambiente. (1991).

Ratzel, F. (1975). "Ubicación y espacio”. En Rattenbach, A. (Ed.), Antología Geopolítica (pp. 15-51). Buenos Aires: Editorial Pleamar.

Roura, R. (2013) El debate sobre áreas marinas protegidas en la Antártida: ¿conservación o pesca? Ecología política (46): 48-56.

Russell, R. (1995). "Políticas exteriores: hacia una política común”. En Rapoport, M. (Ed.). Argentina y Brasil en el MERCOSUR. Políticas comunes y alianzas regionales (pp. 34-44). Buenos Aires: GEL.

Sampaio, D. P. (2015). O Sistema do Tratado Antártico: a consolidação de um regime internacional e o debate sobre seu déficit democrático. Publicação da Associação Brasileira de Relações Internacionais, 10 (1): 83-104.

Sepúlveda Cox, J. (2008). Importancia Geopolítica del Continente Antártico. En Antártica Chilena: presente y futuro. Seminario llevado adelante en la Universidad Andrés Bello, Chile.

Storni, S. (1967). Intereses Argentinos en el mar. Argentina: Instituto de publicaciones navales.

The observatory of economic complexity. (2016). Falkland Islands Trade. Recuperado de https://atlas.media.mit.edu/en/profile/country/flk/.

Tratado Antártico (1959).

Tratado para la Proscripción de Armas Nucleares en América Latina y el Caribe (1969).

Urbina Paredes, J. (2009). El Tratado Antártico, posición de Chile como país puente. UNISCI Discussion Papers, 21: 138-147. 
